\title{
Effusion Indicator
}

National Cancer Institute

\section{Source}

National Cancer Institute. Effusion Indicator. NCI Thesaurus. Code C161422.

An indication as to whether effusion is present. 